Revue internationale P.M.E.

Économie et gestion de la petite et moyenne entreprise

\title{
Les stratégies d'échanges technologiques des petites entreprises industrielles
}

\section{Catherine Belotti}

Volume 13, numéro 2, 2000

URI : https://id.erudit.org/iderudit/1008676ar

DOI : https://doi.org/10.7202/1008676ar

Aller au sommaire du numéro

Éditeur(s)

Presses de l’Université du Québec

ISSN

0776-5436 (imprimé)

1918-9699 (numérique)

Découvrir la revue

Citer cette note

Belotti, C. (2000). Les stratégies d'échanges technologiques des petites entreprises industrielles. Revue internationale P.M.E., 13(2), 75-96. https://doi.org/10.7202/1008676ar
Résumé de l'article

Nous rendons compte d'une enquête menée auprès de petites entreprise suédoises du bois et de l'imprimerie, pour étudier différents types de stratégies d'échanges technologiques développées par ces PMI et définir la logique qui les sous-tend. Les stratégies à succès se fondent sur des échanges élargis avec différents types d'acteurs et sur une logique de proximité organisationnelle. Les organisations professionnelles créent de nombreux « espaces » d'échanges technologiques jugés importants par les entreprises pour leur développement technologique parce qu'elles y expérimentent de nombreuses dimensions de proximité. 


\title{
Notes de recherche
}

\section{Les stratégies d'échanges technologiques des petites entreprises industrielles}

Catherine BELOTTI

Université de Montpellier I

\section{MOTS CLÉS}

\section{PMI - Échanges technologiques \\ Modernisation technologique - Industrie suédoise}

\begin{abstract}
RÉSUMÉ
Nous rendons compte d'une enquête menée auprès de petites entreprise suédoises du bois et de l'imprimerie, pour étudier différents types de stratégies d'échanges technologiques développées par ces PMI et définir la logique qui les sous-tend. Les stratégies à succès se fondent sur des échanges élargis avec différents types d'acteurs et sur une logique de proximité organisationnelle. Les organisations professionnelles créent de nombreux "espaces " d'échanges technologiques jugés importants par les entreprises pour leur développement technologique parce qu'elles y expérimentent de nombreuses dimensions de proximité.
\end{abstract}

\section{L'AUTEURE}

CATHERINE Belottı détient un doctorat en sciences de gestion. Elle est chercheure associée à I'IMIT (Institute for Management of Innovation and Technology) de Stockholm. Ses activités de recherche portent principalement sur les stratégies de mobilisation de connaissances technologiques des PMI et sur les problèmes relatifs à la diffusion de nouvelles technologies dans les PMI. Entre septembre 1998 et décembre 1999, elle a été invitée à l'ERFI, Université de Montpellier I.

\section{REMERCIEMENTS}

L'étude présentée a été financée par la Fondation Jan Wallander, Handelsbanken, Stockholm. L'auteure remercie le professeur Michel Marchesnay, ERFI, Montpellier, pour ses remarques et suggestions sur cet article. 


\begin{abstract}
We present the results of a survey of small wood-working and printing entreprises in Sweden, exploring their patterns of technological exchange in relation with their technological and competence level. Successful exchange patterns show a large variation of exchanges with different kinds of partners and are based on a "logic of proximity". Professional organizations offer many spaces of technological exchange for SMEs where they experiment many proximity dimensions.
\end{abstract}

\title{
RESUMEN
}

Este trabajo presenta los resultados de una encuesta realizada a un grupo de pequeñas empresas suecas del sector de la madera y industria de la imprenta, con el objetivo de estudiar los diferentes tipos de estrategias de cambios tecnológicos desarrollados por estas PyMES y definir la lógica que está subyacente a ellas. Las estrategias de suceso están basadas en cambios ampliados a diferentes tipos de actores y también a una lógica de proximidad entre organizaciones. Las organizaciones profesionales crean numerosos "espacios" de cambios tecnológicos considerados importantes por las empresas para su desarrollo tecnológico, porque estas experimentan, ahí, numerosas maneras de proximidad.

\section{ZUSAMMENFASSUNG}

Wir nehmen Bezug auf eine Untersuchung bei kleinen schwedischen Holz- und Druckereibetrieben, um die verschiedenen Strategieformen für den Technologieaustausch zu untersuchen, welche von diesen kleinen und mittleren Industrieunternehmungen entwickelt wurden. Ziel ist es, die den Strategieformen unterstellte Logik zu definieren.

Die Erfolgsstrategien basieren auf dem breiten Austausch mit verschiedenen Partnern und auf der Logik der organisatorischen Nähe. Die professionellen Organisationen errichten verschiedene technologische Austauschräume, die als wichtig erachtet werden von den Unternehmungen für ihre technische Entwicklung, weil sie stark mit verschiedenen Dimensionen der Annäherung experimentieren.

\section{Introduction}

Il est aujourd'hui bien établi que l'adoption et l'exploitation continuelles de nouvelles technologies sont des facteurs stratégiques de première importance pour les petites entreprises industrielles qui doivent faire face à de multiples demandes d'amélioration de leurs produits et services de la part de leurs clients. Durant les 20 dernières années, de nombreuses études ont cherché à mieux cerner les conditions facilitant le développement technologique de ces PMI, que ce soit en étudiant les facteurs internes favorisant l'adoption de nouvelles technologies, les caractéristiques des processus d'implantation ou le rôle de l'environnement dans le développement technologique des PMI. Ainsi, il a été clairement démontré que les caractéristiques du dirigeant, particulièrement son niveau de formation et la qualité de sa veille 
technologique, étaient des facteurs importants de développement technologique dans l'entreprise. Le caractère interactif de ce développement a été par ailleurs largement souligné.

Les initiatives de diffusion technologique en direction des petites entreprises industrielles, qui se sont multipliées au rythme de l'importance croissante donnée par les politiques industrielles aux PME, ont su, à première vue, tirer les leçons de cette recherche. En effet, tout en se diversifiant, elles se sont recentrées sur deux axes, le transfert dans les PME de capital humain ayant une formation supérieure et la création de toutes sortes de réseaux d'échanges ou de développements technologiques favorisant l'interaction de différentes catégories de partenaires - à tel point que l'utilisation du concept de réseau est devenue incontournable dans tout discours de politique de diffusion de technologie. Toutefois, la redondance quelquefois anarchique des programmes ou les difficultés à définir les cibles de différents types d'initiatives nous semblent refléter une connaissance encore limitée des dynamiques propres à différents types d'entreprises et des logiques selon lesquelles elles ont recours à leur environnement et l'utilisent pour soutenir leur renouveau technologique (Belotti, 1997; Belotti et Tunälv, 1999).

L'objectif de l'enquête présentée dans cet article et menée auprès d'entreprises suédoises de l'industrie du bois et de l'imprimerie est de mettre en évidence les différents types de stratégies d'échanges technologiques développées par ces PMI et de définir la logique qui sous-tend ces stratégies, en les reliant aux stratégies de développement des techniques et des compétences des entreprises ainsi qu'aux caractéristiques de leur environnement.

\section{Les fondements et les orientations de la recherche}

Notre représentation du renouveau technologique ayant cours dans les petites entreprises industrielles s'est élaborée à partir de trois grands types d'études empiriques menées dans le domaine. Tout d'abord, un important travail a été effectué, notamment par les chercheurs canadiens, sur les facteurs influençant l'adoption de nouvelles technologies dans les petites entreprises industrielles (Julien et Hébert, 1986 ; Julien, Carrière et Hébert, 1988 ; Julien et al., 1994 ; Julien, 1996). Ces recherches ont établi que le niveau de formation des chefs d'entreprises, leur capacité d'exploitation d'informations spécialisées, incluant la collaboration avec des instances de recherche, étaient les principaux facteurs discriminant les petites entreprises adoptant de nouvelles technologies. Un personnel technique qualifié et une organisation plus complexe constituaient aussi des caractéristiques spécifiques aux petites entreprises les plus actives techniquement.

Un autre type de recherche s'est intéressé aux processus spécifiques d'implantation de nouvelles technologies dans les petites entreprises. Ces travaux ont mis 
en évidence des processus étirés dans le temps, aux dimensions cognitives et sociales complexes, conduisant souvent à des formes inattendues d'exploitation de la technique et requérant fréquemment une interaction de l'entreprise avec différents acteurs externes (Norén, 1987; Langley et Truax, 1994 ; Gasse, 1986, 1995; Mønsted, 1989). Parce que focalisées sur les processus intra-organisationnels, ces recherches ont fourni une connaissance superficielle des interactions de l'entreprise avec l'environnement.

Par contre, un certain nombre d'auteurs ont exploré l'utilisation faite par les PME de différentes sources externes d'informations et de supports relatifs au changement technologique ( $c f$. par exemple Leonard-Barton, 1982 ; Höglund et Persson, 1984 ; Fölster, 1991 ; SBRC, 1992 ; Guedj et Picard, 1994). Les résultats statistiques livrés par ces études sont généraux (et partiellement contradictoires), quelques auteurs fournissant cependant une image plus détaillée des différents types d'informations et de supports utilisés par les entreprises dans les différentes phases d'adoption et d'implantation de nouvelles technologies (Allen, Hyman et Pinkney, 1983 ; Lapointe, 1991). Deux types de conclusions ressortent le plus souvent des analyses de ces recherches. D'une part, l'importance qu'aurait la proximité géographique des partenaires pour les échanges technologiques, postulat fondant le concept des milieux innovateurs (cf. Camagni, 1991), contredit cependant par certains auteurs (Héraud et Nanopoulos, 1994 ; Grotz et Braun, 1996). D'autre part, la fréquence avec laquelle les entreprises indiquent leurs clients ou fournisseurs comme source de changements technologiques dans de nombreuses études a conduit certains auteurs à privilégier les relations clients-fournisseurs dans l'étude des échanges technologiques entre entreprises et à en postuler le rôle prééminent pour le développement technologique ( $c f$. Håkansson, 1989).

Principalement pour des raisons méthodologiques, l'ensemble de ces études se sont centrées sur l'adoption et l'implantation de technologies spécifiques et de pointe, discriminant les entreprises innovantes (qui les adoptent) des entreprises techniquement passives ou suiveuses (qui ne les adoptent pas ou les adoptent plus tard). Pourtant, nous savons que le développement technologique des petites entreprises se fonde la plupart du temps sur une succession de changements incrémentaux (Allen et al., 1983 ; Le Bas et Géniaux, 1994) qui peut engager l'entreprise dans des trajectoires technologiques différentes selon la stratégie de développement qui émerge dans l'entreprise sur le long terme. Il est donc raisonnable de penser que c'est d'après le profil technologique général des petites entreprises, plutôt que l'adoption d'une technologie spécifique de pointe, que nous devons juger de leur capacité de changement et d'innovation. Ainsi, Rogers (1983) note-t-il que les chefs d'entreprises relient les technologies les unes aux autres lorsqu' ils s'engagent dans des changements technologiques et suggère que l'on tienne compte des ensembles de technologies «technology clusters », plutôt que des projets isolés, pour comprendre le développement technologique qui a cours dans les entreprises. 
Reprenant la suggestion de Rogers (1983) à notre compte, nous avons étudié sur quelques cas d'entreprises, la succession, sur le long terme, des changements technologiques opérés dans des PMI de transformation du bois (Belotti, 1996, 1999a et b). Ces études de cas ont montré comment les entreprises technologiquement actives, sur le long terme, diversifiaient leur mode d'échanges technologiques avec l'environnement et complexifiaient en même temps leur organisation pour créer des synergies entre différentes sortes de relations. En d'autres termes, elles n'ont pas abouti à discerner un type de relation privilégiée pour le développement technologique des PMI, mais mis en évidence une dynamique opérant dans le temps entre le processus de changement technologique dans l'entreprise, le développement de la compétence de l'entreprise et la diversification de ses échanges technologiques. Ces trois éléments nous paraissent être les piliers constitutifs et interdépendants de la stratégie des petites entreprises en matière technologique, qu'il nous faut essayer de cerner pour comprendre ces stratégies. Ils opérationnalisent d'ailleurs en la matière les éléments fondamentaux (activité, organisation, environnement) du modèle de management stratégique développé et utilisé par Marchesnay (1994) à propos des petites entreprises.

L'enquête présentée ci-dessous prolonge l'approche que nous avons adoptée dans nos études de cas antérieures et vise à en confirmer, voire à affiner, certaines conclusions auprès d'un plus large échantillon d'entreprises. Voici quels étaient ses objectifs :

- explorer les relations existant entre a) le niveau technologique des petites entreprises, $b$ ) leur niveau de compétence et leur activité de développement de ces compétences et $c$ ) leur mode d'échanges technologiques avec l'environnement ;

- identifier différentes stratégies d'échange, associées à différents niveaux technologiques et de compétence des entreprises ;

- développer, à la lumière des résultats et au regard de nos études qualitatives antérieures, une interprétation de ces stratégies et des logiques qui les sous-tendent.

La spécificité de cette étude réside dans notre ambition à cerner, dans le cadre d'une méthode d'enquête, la dynamique qui se crée dans le temps entre l'entreprise et son environnement concernant son développement technologique.

\section{L'étude empirique}

L'étude empirique a été effectuée parmi des entreprises de l'industrie de deuxième transformation du bois et de l'imprimerie. Nous nous sommes limitée à deux industries afin d'être en mesure de répertorier en détail, dans chaque cas, les différentes 
technologies utilisées par les entreprises et le système technologique propre à chaque industrie, ce qui requérait des phases d'étude qualitative préliminaires pour chaque industrie. L'industrie du bois a été choisie comme constituant un secteur d'activité important de l'industrie suédoise, que nous connaissons bien et qui, à l'inverse de l'imprimerie, n'a pas eu à faire face à des changements technologiques radicaux durant les dernières décennies. La technologie desk-top et la digitalisation des procédés de production graphique ont, en revanche, radicalement transformé les conditions de concurrence des petites imprimeries et la structure du système technologique auquel elles se réfèrent. Nous avons fait l'hypothèse que ce contraste pouvait mettre en évidence des différences intéressantes dans les stratégies des firmes pour mobiliser et développer des connaissances technologiques.

\subsection{Méthodologie}

Nous avons constitué, à partir du Fichier des entreprises du bureau suédois des statistiques (SCB-företagsregister) un échantillon aléatoire de 172 entreprises indépendantes et comprenant entre 10 et 49 employés, également réparties entre l'industrie de deuxième transformation du bois et celle de l'imprimerie. Les dirigeants des entreprises sélectionnées ont été contactés par téléphone pour un entretien d'environ un quart d'heure. Les 132 dirigeants ayant accepté de participer à cet entretien téléphonique ont ensuite reçu par courrier un questionnaire supplémentaire qui a été retourné par 117 entreprises (ce qui représente un taux final de réponse de $68 \%^{1}$ ). Ces 117 entreprises représentent $37,5 \%$ de la population totale des PMI suédoises indépendantes et comprenant 10 à 49 employés dans les deux industries étudiées. Le tableau 1 montre leur répartition au regard de la taille.

\section{TABLEAU 1 \\ Structure de l'échantillon par rapport au nombre d'employés dans les entreprises}

\begin{tabular}{lcc}
\hline & Bois $(\boldsymbol{n}=\mathbf{5 4})$ & Imprimerie $(\boldsymbol{n}=\mathbf{6 3})$ \\
\hline Nombre moyen d'employés & 18,2 & 17,9 \\
\hline Pourcentage d'entreprises avec & $72,2 \%$ & $76,2 \%$ \\
$-10-19$ employés & $27,8 \%$ & $23,8 \%$ \\
$-20-50$ employés & & \\
\hline
\end{tabular}

Les deux questionnaires couvraient ensemble quatre domaines d'investigation des entreprises :

1. Ce résultat qui, jugé à l'aune de l'expérience de nombreux pays, peut paraître impressionnant est un résultat normal pour la Suède où les entreprises, par tradition, sont très disponibles pour les enquêtes et études (Holmquist et Sundin, 1997). 
- leurs caractéristiques structurelles et commerciales (taille, croissance, export, structure des relations commerciales);

- leur niveau de compétence (niveau de formation du dirigeant et des cadres, degré de diversification des formations, degré de différenciation fonctionnelle de l'organisation) et leur stratégie de développement de compétence (recrutement de cadres et activités de formation durant les deux dernières années, objectifs et priorités donnés aux activités de formation);

- leurs échanges technologiques avec l'environnement. Cette partie du questionnaire a largement bénéficié de l'étude qualitative que nous avons menée en début de projet pour identifier et caractériser le «système technologique » (Carlsson et Stanckiewicz, 1991), c'est-à-dire l'environnement institutionnel pertinent dont on doit tenir compte dans chaque industrie (19 entrevues et la consultation d'un large éventail de documents institutionnels ou scientifiques relatifs à ces systèmes). La plupart des variables étudiées apparaissent dans les tableaux résumant les résultats de l'étude, présentés plus loin;

- leur niveau technique (mesuré à partir des différentes techniques de production et de gestion présentes dans l'entreprise) et leur stratégie de développement technologique (plans d'investissement en nouvelles technologies et objectifs liés à ces plans ; éventuelles barrières à l'introduction de nouvelles technologies). Le niveau technique a été mesuré par un «index technique » calculé en attribuant des valeurs pondérées aux différentes techniques présentes dans l'entreprise, la valeur maximale de l'index d'une entreprise pouvant être de 100. Cet index a été construit de façon ad hoc pour chaque industrie en étroite collaboration avec trois experts des technologies de l'imprimerie et deux experts des technologies de transformation du bois. La pondération des variables a tenu compte du fait que certaines techniques pouvaient en exclure d'autres, selon les choix stratégiques des entreprises.

Cet index a été validé en deux temps. Tout d'abord, avant l'enquête, le questionnaire a été prétesté dans quatre entreprises, pour vérifier si les questions concernant les techniques étaient jugées pertinentes par les entrepreneurs et décrivaient bien leur situation technique dans son ensemble. Ce prétest a seulement engendré quelques modifications dans la formulation des questions. Ensuite, une fois les données recueillies, les valeurs de l'index technique obtenues ne reposant pas sur les mêmes variables dans les entreprises de chaque industrie, nous avons transformé cette valeur en trois « niveaux technologiques » afin de pouvoir analyser les données à l'intérieur de tout l'échantillon : haut niveau technologique pour l'industrie (NTH, quartile supérieur des valeurs de chaque industrie); niveau technologique bas (NTB, quartile inférieur); niveau technologique moyen (NTM, quartile intermédiaire). 
Nous avons alors comparé le classement de chaque entreprise avec le classement fait par le dirigeant lui-même en réponse à l'une des questions posées à ce sujet. Cette comparaison n'a pas donné lieu à une remise en question du classement obtenu à partir de nos valeurs d'index.

\section{Les principaux résultats de l'enquête}

\subsection{Implantation de nouvelles techniques et compétence}

Il faut d'abord souligner que le niveau moyen d'implantation des techniques modernes de production et de gestion est relativement élevé dans les entreprises étudiées et que seules les techniques vraiment de pointe comme, par exemple, les presses digitales dans l'imprimerie ou des systèmes de production totalement intégrés dans le bois restent rares, touchant moins de $10 \%$ des entreprises étudiées. Dans les deux branches, la mise en place d'un flux de production plus efficace reste l'objectif principal lié à de nouveaux investissements. Signalons cependant que les deux industries se distinguent considérablement en ce qui concerne les plans et stratégies d'investissement en nouvelles techniques. L'ensemble des imprimeries qui n'ont pas introduit de nouvelles techniques ces deux dernières années ont prévu dans leur budget d'investir dans le court terme. Le renforcement de la relation client et l'image de la firme sont les principaux aspects invoqués par les imprimeries pour justifier leurs projets d'investissement. Par contre, moins de la moitié des manufactures du bois ont inscrit l'implantation de nouvelles techniques dans leur prochain budget, un tiers des entreprises prétextant des barrières économiques à l'investissement. La baisse des coûts de production et la flexibilité en termes de volume de séries sont les aspects auxquels les entreprises du bois donnent une large importance pour justifier leurs projets d'investissement. Notons enfin que, dans les deux branches, une mince proportion d'entreprises relient leurs plans d'investissement en nouvelles techniques à des objectifs de développement de nouveaux produits.

Le niveau moyen de formation des dirigeants est plutôt élevé dans les deux branches, contrairement à l'image que l'on a souvent des PME à ce sujet. Le quart des chefs d'entreprises du bois et $32 \%$ des dirigeants d'imprimerie ont une formation supérieure. De même, plus de la moitié des imprimeries et le tiers des manufactures du bois ont au moins un cadre ayant une formation universitaire ou supérieure. De la même manière, les entreprises des deux branches sont en moyenne plutôt actives pour développer les compétences de leur personnel par le biais de la formation continue ${ }^{2}$. Seulement $21 \%$ d'entre elles déclarent ne pas avoir organisé

2. Il faut noter que la formation continue en Suède ne fait pas l'objet de contraintes légales comme c'est le cas par cxemple en France. 
de formation continue de leur personnel durant les dernières années, alors que plus de la moitié des entreprises rapportent avoir à la fois organisé des stages à l'intérieur de l'entreprise et envoyé leurs employés suivre des cours externes.

\subsection{L'échanges technologiques avec les clients et les fournisseurs}

Comme on peut le constater à la lecture du tableau 2, une large proportion d'entreprises déclarent collaborer avec leurs clients et leurs fournisseurs de matières premières ou de services pour développer leurs procédés ou leurs produits. Cependant, nous ne trouvons pas de relation statistiquement significative entre le fait que les entreprises coopèrent avec leurs clients et fournisseurs pour le développement de produits et procédés et le niveau technologique des entreprises.

TABLEAU 2

Les échanges technologiques des petites entreprises avec leurs clients et leurs fournisseurs de matières premières et services, par industrie et par niveau technologique

\begin{tabular}{lccccccc}
\hline $\begin{array}{l}\text { Pourcentage } \\
\text { de firmes/d'entrepreneurs }\end{array}$ & $\begin{array}{c}\text { Imprimerie } \\
(\boldsymbol{n}=\mathbf{6 3})\end{array}$ & $\begin{array}{c}\text { Bois } \\
(\boldsymbol{n}=\mathbf{5 4})\end{array}$ & Sign & NTH & NTM & NTB & Sign \\
\hline Coopérant avec & & & & & & & \\
- - les clients sur les produits & 47 & 83 & $* * *$ & 71 & 62 & 61 & \\
- les clients sur les procédés & 24 & 17 & & 29 & 18 & 18 & \\
- les fournisseurs & 30 & 57 & $* * *$ & 50 & 43 & 36 & \\
\hline Ayant eu des demandes technologiques & & & & & & & \\
$-\quad$ de certains clients & 46 & 33 & & 50 & 42 & 25 & \\
- de certains fournisseurs & 8 & 9 & & 7 & 12 & 4 & \\
\hline Ayant influencé technologiquement & & & & & & & \\
- certains clients & 75 & 9 & $* * *$ & 64 & 56 & 43 & \\
- certains fournisseurs & 41 & 44 & & 57 & 43 & 29 & \\
\hline
\end{tabular}

Note: Sign. ANOVA $* \mathrm{p}<0,1,{ }^{* *} \mathrm{p}<0,05,{ }^{* * *} \mathrm{p}<0,01$.

C'est entre les entreprises de chaque industrie que l'on trouve des différences statistiquement significatives concernant les échanges technologiques avec les clients et les fournisseurs. Elles montrent que le changement technologique est influencé par les demandes des clients dans le bois et par la poussée de la technologie dans l'imprimerie. En effet, les entreprises du bois rapportent en proportion significativement plus large qu'elles collaborent avec leurs clients. Celles qui ont de plus gros clients collaborent davantage avec leurs clients et la collaboration avec les clients est associée de façon significative à la collaboration avec les fournisseurs. Les entreprises du bois attribuent plus d'importance que leurs homologues de l'imprimerie à leurs clients, s'agissant d'obtenir des informations et des idées sur les nouvelles technologies. Elles donnent aussi une plus grande importance, lors 
de leurs investissements techniques, aux objectifs de diminution des coûts et de flexibilité dans les volumes de production - reflétant les demandes des clients dans ces domaines.

Les imprimeries, quant à elles, ne rapportent pas une collaboration aussi étendue avec leurs clients et leurs fournisseurs mais sont significativement plus nombreuses à estimer qu'elles ont influencé certains de leurs clients dans l'adoption de nouvelles technologies. Du côté de leurs fournisseurs, une grande concurrence parmi leurs prestataires de services reprographiques explique qu'elles préfèrent changer de prestataire lorsqu'il ne répond pas technologiquement à leurs exigences plutôt que de consacrer des efforts à améliorer leurs échanges d'informations techniques. On peut ainsi penser que la poussée technologique qu'ont connue ces entreprises, notamment la digitalisation de leurs procédés, a créé de nouvelles relations de pouvoir, dépendance et concurrence le long de la chaîne de valeur, laissant des marges de manœuvre aux imprimeries les plus proactives pour mener l'intégration technologique de leurs partenaires commerciaux. De fait, et s'agissant des investissements en nouvelles technologies, les imprimeries accordent le plus d'importance aux objectifs de s'attacher certains clients et d'acquérir une image d'entreprise technologiquement avancée.

TABLEAU 3

Les échanges technologiques des petites entreprises avec leurs équipementiers, par industrie et par niveau technologique

\begin{tabular}{lccccccc}
\hline $\begin{array}{l}\text { Pourcentage } \\
\text { de firmes /d'entrepreneurs }\end{array}$ & $\begin{array}{c}\text { Imprimerie } \\
(\boldsymbol{n}=\mathbf{6 3})\end{array}$ & $\begin{array}{c}\text { Bois } \\
(\boldsymbol{n}=\mathbf{5 4})\end{array}$ & Sign & NTH & NTM & NTB & Sign \\
\hline Fidèles aux équipementiers & 60 & 30 & $* *$ & 73 & 48 & 46 & \\
\hline Coopérant avec les équipementiers & 36 & 59 & $* *$ & 57 & 49 & 32 & \\
\hline $\begin{array}{l}\text { Informées régulièrement } \\
\text { par les équipementiers }\end{array}$ & 86 & 98 & $* *$ & 86 & 92 & 96 & \\
\hline $\begin{array}{l}\text { Visitant régulièrement } \\
\text { les foires techniques }\end{array}$ & & & & & & & \\
$\begin{array}{l}- \text { nationales } \\
- \text { internationales }\end{array}$ & 79 & 68 & & 89 & 71 & 68 & $* * *$ \\
\hline Ayant testé des prototypes & 56 & 33 & $* *$ & 75 & 38 & 32 & $* * *$ \\
\hline $\begin{array}{l}\text { Achetant de la formation } \\
\text { aux équipementiers }\end{array}$ & 72 & 79 & & 89 & 80 & 50 & $* * *$ \\
\hline
\end{tabular}

Note : Sign. ANOVA $* \mathrm{p}<0,1,{ }^{* *} \mathrm{p}<0,05,{ }^{* * *} \mathrm{p}<0,01$.

En ce qui concerne les échanges avec les équipementiers, on constate aussi dans le tableau 3 des différences significatives entre les deux industries. Les entreprises du bois se disent en plus grand nombre être informées régulièrement par leurs 
équipementiers, collaborer avec eux lors de l'implantation de nouvelles techniques mais sans pour cela être fidèles à certains d'entre eux lors de nouveaux investissements. C'est par contre le cas des imprimeries, qui ne collaborent pourtant pas en aussi grand nombre avec leurs équipementiers pour installer de nouveaux équipements.

Nous expliquons ces différences par les diverses conditions de formation des nouvelles technologies dans les deux industries. Le développement technologique dans le bois est toujours fortement associé au développement du savoir-faire dans la production et fondé sur une collaboration industrie-équipementiers dans le cadre de changements progressifs des procédés de production, qui laissent aux firmes une certaine flexibilité s'agissant des types de développement ultérieurs. La digitalisation des procédés dans l'imprimerie a par contre impliqué un déplacement des activités de développement technologique chez les grands fournisseurs de systèmes « soft » et « hard » qui fournissent des systèmes standardisés et adaptés à la production à petite échelle, ce qui rend les entreprises plus dépendantes de leurs fournisseurs une fois qu'elles ont choisi un type de système - à moins qu'elles ne développent une compétence interne de R-D. De fait, les imprimeries donnent la plus grande importance aux revues techniques puis à leurs équipementiers en ce qui concerne l'acquisition d'idées et d'information sur les nouvelles technologies. Elles sont significativement plus nombreuses que dans le bois à visiter les foires techniques internationales, à tester des prototypes (le plus souvent des versions beta de systèmes) et à acheter de la formation à leurs équipementiers. Dix pour cent d'entre elles désignent leur personnel et leur propre activité de développement comme sources importantes de nouvelles technologies alors qu' aucune des entreprises du bois ne rapporte une telle activité de R-D.

\subsection{Les échanges technologiques avec d'autres petites entreprises du même secteur}

Une très large proportion de chefs d'entreprises déclarent avoir régulièrement des échanges avec des collègues d'autres petites entreprises sur des enjeux technologiques, et ce, surtout dans l'industrie du bois. Sur ce point, nous ne trouvons pas de différences très significatives entre entreprises de niveaux technologiques différents. Beaucoup d'entreprises déclarent aussi être membres d'un réseau formalisé d'entreprises, c'est-à-dire ayant une structure juridique, se référant au total à 30 réseaux différents dont 19 dans l'industrie du bois. Quarante pour cent des entreprises déclarent recevoir la visite d'autres entreprises au moins une ou deux fois par an, souvent en liaison avec l'implantation de nouveaux équipements, et plus de $45 \%$ des chefs d'entreprises déclarent avoir eu l'occasion de communiquer leur expérience en matière de technologie à d'autres petites entreprises. La fréquence de cette activité de «diffusion technologique » est significativement plus élevée dans les entreprises à haut niveau technologique que dans les autres. 
TABLEAU 4

Les échanges technologiques des petites entreprises avec d'autres petites entreprises, par industrie et par niveau technologique

\begin{tabular}{lccccc}
\hline $\begin{array}{l}\text { Pourcentage } \\
\text { de firmes/d'entrepreneurs }\end{array}$ & $\begin{array}{c}\text { Imprimerie } \\
(n=63)\end{array}$ & $\begin{array}{c}\text { Bois } \\
(n=54)\end{array}$ & Sign NTH NTM NTB Sign \\
\hline
\end{tabular}

Ayant des échanges technologiques

avec d'autres PMI dans le cadre de

- contacts personnels informels

- réunions professionnelles

- réunions locales

- réseau formel d'entreprises

$\begin{array}{lllllll}70 & 88 & * * & 93 & 73 & 71 & * \\ 43 & 51 & & 46 & 50 & 37 & \\ 16 & 45 & * * & 25 & 33 & 21 & \\ 26 & 53 & * * * & 39 & 42 & 25 & \end{array}$

Ayant très souvent des visites

d'autres PMI 16

$16 \quad 32 \quad * \quad 43 \quad 20 \quad 11 \quad * * *$

Ayant donné des conférences

sur des sujets techniques

à d'autres PMI

49

44

$75 \quad 43$

$29 \quad * * *$

Note : Sign. ANOVA $* \mathrm{p}<0,1,{ }^{* *} \mathrm{p}<0,05, * * * \mathrm{p}<0,01$.

\subsection{L'utilisation de sources de connaissances privées ou institutionnelles}

Le tableau 5 indique que plus d'un tiers des entreprises déclarent avoir utilisé les services d'un consultant privé en liaison avec l'adoption de nouvelles techniques ou l'achat de nouveaux ordinateurs ou logiciels. Mais un résultat plus inattendu est la fréquence relativement élevée à laquelle les entreprises déclarent avoir recours aux instituts de recherches et aux universités. Cette fréquence de contact avec la recherche s'apparente plutôt à celles rapportées antérieurement concernant les entreprises de haute technologie (Beesley et Rothwell, 1987 ; Rothwell et Dodgson, 1991). Les résultats concordent toutefois avec ceux d'un récent projet européen sur les PMI et les technologies d'information dont l'enquête menée auprès de plus de 500 PMI de tous secteurs en Suède contenait une question sur leur recours aux sources de connaissance privées et institutionnelles ( $c f$. Belotti et Tunälv, 1998). Cela met en question l'idée commune depuis longtemps bien établie (du moins en Suède) qu'il existe de fortes barrières aux échanges entre entreprises et institutions de recherche (Håkansson, 1989). Nous pouvons penser que ces nouveaux résultats reflètent l'impact réel d'une politique offensive, durant ces dernières années, de contact avec les entreprises de la part des institutions de recherche. Ils peuvent aussi indirectement refléter l'accroissement du niveau de formation des dirigeants et cadres - ceux d'entre eux, de plus en plus nombreux, à avoir une formation de niveau universitaire ayant plus de facilité à s'adresser à ce milieu où ils ont même quelquefois créé des contacts personnels. 
TABLEAU 5

Les échanges technologiques des petites entreprises avec différentes sources de connaissances privées ou institutionnelles, par industrie et par niveau technologique

\begin{tabular}{lccrrrrr}
\hline $\begin{array}{l}\text { Pourcentage } \\
\text { de firmes/d'entrepreneurs }\end{array}$ & $\begin{array}{c}\text { Imprimerie } \\
(\boldsymbol{n}=\mathbf{6 3})\end{array}$ & $\begin{array}{c}\text { Bois } \\
(\boldsymbol{n}=\mathbf{5 4})\end{array}$ & Sign & NTH & NTM & NTB & Sign \\
\hline $\begin{array}{l}\text { Ayant utilisé le support de } \\
\text { - conseillers techniques privés }\end{array}$ & 40 & 37 & & 43 & 44 & 22 & \\
- institut de recherche industrielle & & & & & & & \\
$\quad$ de la branche & 48 & 65 & $* *$ & 82 & 55 & 28 & $* * *$ \\
- université nationale & 17 & 17 & & 25 & 16 & 11 & \\
- conseiller public local & 10 & 21 & $*$ & 25 & 14 & 3 & $*$ \\
- université étrangère & 4 & 4 & & 11 & 3 & 0 & \\
- institut de recherche industrielle & & & & & & & \\
$\quad$ hors branche & 6 & 3 & & 4 & 17 & 12 & \\
\hline
\end{tabular}

Note : Sign. ANOVA $* \mathrm{p}<0,1,{ }^{* *} \mathrm{p}<0,05,{ }^{* * *} \mathrm{p}<0,01$.

Le recours aux consultants privés et aux organisations de recherche ne varie pas d'une branche à l'autre mais est significativement plus étendu parmi les entreprises ayant un haut niveau technologique que parmi les autres entreprises. En même temps, nous pouvons constater que les entreprises ont recours dans une mesure limitée aux organisations de conseil public locales.

Un autre aspect mis en lumière par l'enquête est le rôle que les entrepreneurs attribuent à leurs associations professionnelles en matière de diffusion et de soutien au développement technologique. Les associations professionnelles sont une catégorie d'acteurs très largement citée ( $55,6 \%$ des entreprises) comme source d'idées et d'informations sur les nouvelles technologies à travers les activités qu'elles organisent. Ce résultat est cohérent avec la fréquence à laquelle les entreprises déclarent avoir des échanges technologiques avec d'autres petites entreprises dans le cadre d'activités associatives professionnelles, ou celle à laquelle elles déclarent participer aux séminaires d'information technologique organisés par ces associations. Il faut aussi noter que les journaux professionnels sont la source d'idées et d'informations sur les nouvelles technologies la plus largement citée (87\% des entreprises) et que les journaux les plus largement lus par les entrepreneurs étudiés sont justement édités par ces associations. Enfin, les entrepreneurs dont les entreprises ont un haut niveau technologique déclarent avoir des engagements formels au sein de leur association professionnelle à une fréquence largement et significativement plus élevée que les autres entrepreneurs. 


\section{Les stratégies d'échanges technologiques des entreprises}

Dans une deuxième étape de notre analyse, nous avons essayé de déterminer, à l'aide d'une procédure d'analyse typologique de nos données, si les entreprises avaient différentes façons de combiner leurs recours à ces différentes sources d'informations et de supports technologiques. Nous avons limité l'analyse typologique à 14 variables décrivant les échanges technologiques des entreprises avec différentes catégories d'acteurs. Ces variables ont été choisies de façon à couvrir l'ensemble des catégories de sources d'information et support étant utilisées par les entreprises et apparaissant importantes dans l'analyse préliminaire. Nous avons retenu l'analyse en quatre groupes comme donnant des groupes bien distinctifs par rapport aux variables prises en considération et discriminantes dans chaque catégorie d'acteur (tableau 6). Ces groupes, une fois identifiés, ont été examinés sous l'angle du niveau technologique des entreprises qui les composent ainsi que des différentes variables d'environnement et des compétences étudiées dans l'enquête afin de mieux les caractériser sur le plan des stratégies d'échanges (tableau 7).

TABLEAU 6

Résultat de l'analyse typologique des échanges technologiques des entreprises

\begin{tabular}{|c|c|c|c|c|}
\hline $\begin{array}{l}\text { Variables d'échanges } \\
\text { avec les partenaires }\end{array}$ & Gr. 1 & Gr. 2 & Gr. 3 & Gr. 4 \\
\hline Coopération clients sur les produits $* * *$ & 1,04 & 1,91 & 1,50 & 1,79 \\
\hline Coopération clients sur les procédés $* * *$ & 1,00 & 1,46 & 1.25 & 1.15 \\
\hline Coopération fournisseurs $* * *$ & 1,08 & 1,43 & 1,38 & 1,68 \\
\hline Coopération équipementiers * & 1,25 & 1,54 & 1,50 & 1,56 \\
\hline Fidélité aux équipementiers & 1,63 & 1,34 & 1,44 & 1,50 \\
\hline Échanges informels dans autres PMI *** & 1,54 & 1,80 & 1,63 & 1,97 \\
\hline Échanges dans un réseau formel de PMI & 1,25 & 1,46 & 1,44 & 1,35 \\
\hline $\begin{array}{l}\text { Utilisation de conseillers techniques } \\
\text { privés } * * *\end{array}$ & 1,25 & 1,46 & 1,69 & 1,29 \\
\hline Utilisation de conseillers publics locaux & 1,08 & 1,11 & 1,25 & 1,15 \\
\hline $\begin{array}{l}\text { Utilisation d'instituts de recherche } \\
\text { industrielle de branche } \\
* * *\end{array}$ & 0,04 & 0,26 & 1,63 & 1,35 \\
\hline $\begin{array}{l}\text { Utilisation d'instituts de recherche } \\
\text { industrielle hors branche }\end{array}$ & 1,00 & 1,03 & 1,03 & 1,06 \\
\hline Utilisation d'université nationale $* * *$ & 1,12 & 1,11 & 1,50 & 1,12 \\
\hline Utilisation d'université internationale & 1,00 & 1,03 & 1,13 & 1,06 \\
\hline $\begin{array}{l}\text { Engagement organisations } \\
\text { professionnelles } * * *\end{array}$ & 0,13 & 0,14 & 1,75 & 0,32 \\
\hline
\end{tabular}

Note : Sign. ANOVA, ${ }^{*} \mathrm{p}<0,1,{ }^{* *} \mathrm{p}<0,05,{ }^{* * *} \mathrm{p}<0,01$. 


\subsection{Mode d'échanges dépendant des fournisseurs d'équipement (groupe 1)}

Un premier groupe d'entreprises n'a pas beaucoup de coopération avec leurs clients et fournisseurs ni avec d'autres petites entreprises. Un petit nombre d'entre elles coopèrent avec les équipementiers auxquels elles sont fidèles ; c'est le cas du plus grand nombre. Elles n'ont pas recours aux instituts de recherche et un très petit nombre d'entre elles ont recours aux autres agents de diffusion technologique. Elles n'ont dans l'ensemble quasiment pas d'échanges technologiques avec les sources privées ou institutionnelles de connaissance. Plutôt que de parler de «stratégie» d'échange, nous pouvons parler pour ces entreprises d'un mode de développement technologique dépendant des fournisseurs d'équipements.

\section{TABLEAU 7}

\section{Caractéristiques structurelles, technologiques et de compétence des différents groupes stratégiques}

\begin{tabular}{|c|c|c|c|c|}
\hline $\begin{array}{l}\text { Caractéristiques } \\
\text { des groupes }\end{array}$ & Gr. 1 & Gr. 2 & Gr. 3 & Gr. 4 \\
\hline $\begin{array}{l}\text { Nombre d'entreprises } \\
\text { - imprimerie } \\
\text { - bois } \\
\text { Nombre moyen d'employés } * * * \\
\text { Niveau technologique moyen }(0-2) * * * \\
\text { Nombre moyen de sources hors marché } \\
\text { et entreprises } * * *\end{array}$ & $\begin{array}{r}24 \\
19 \\
5 \\
12,8 \\
0,54 \\
0,50\end{array}$ & $\begin{array}{c}35 \\
19 \\
16 \\
17,2 \\
1,06 \\
1,00\end{array}$ & $\begin{array}{r}16 \\
9 \\
7 \\
24,3 \\
1,38 \\
2,63\end{array}$ & $\begin{array}{c}34 \\
14 \\
20 \\
20,1 \\
1,24 \\
1,68\end{array}$ \\
\hline $\begin{array}{l}\text { Niveau formel de formation - } \\
\text { entrepreneur }(1-5) \\
\text { Niveau formel de formation - } \\
\text { cadres }(1-3) \\
\text { Diversification formation - } \\
\text { entrepreneur et cadres }(1-3) * * \\
\text { Pourcentage d'entreprises avec plus } \\
\text { de trois fonctions de management }\end{array}$ & $\begin{array}{l}3,18 \\
1,85 \\
1,65\end{array}$ & $\begin{array}{l}1,84 \\
1,38\end{array}$ & $\begin{array}{l}2,06 \\
1,61\end{array}$ & $\begin{array}{l}1,87 \\
1,81\end{array}$ \\
\hline $\begin{array}{l}\text { Pourcentage d'entreprises } \\
\text { organisant formation interne } * * * \\
\text { Pourcentage d'entreprises envoyant } \\
\text { personnel en formation externe } * * * \\
\text { Pourcentage d'entreprises ayant } \\
\text { récemment recruté, nouvelle fonction }\end{array}$ & 37 & 66 & 94 & 74 \\
\hline
\end{tabular}

Note : Sign. ANOVA, ${ }^{*} \mathrm{p}<0,1,{ }^{* *} \mathrm{p}<0,05,{ }^{* * *} \mathrm{p}<0,01$.

Nous observons en même temps un contraste très net entre les entreprises de ce groupe et les autres entreprises en ce qui concerne leur niveau technologique, leur structure et leur niveau de compétence. Ce groupe ne comprend aucune entreprise 
ayant un niveau technologique élevé, mais compte la plus large proportion d'entreprises ayant un bas niveau technologique. Ce sont exclusivement des petites entreprises (moins de 20 employés), qui se différencient des autres groupes par le niveau de formation de leurs cadres qui est bien moins élevé que dans les autres groupes, et surtout par leur activité de développement de compétence qui est significativement moindre que dans les autres groupes. Nous remarquons que la plupart des entreprises de ce groupe sont des imprimeries dont on peut penser qu'elles n'ont pas - à cause d'un manque de ressources financières et de compétence stratégique - pu ou su assumer la poussée technologique observée dans la branche et défendre une position forte au sein de leur réseau traditionnel de clients et de fournisseurs.

\subsection{Stratégie d'échanges élargis, ancrée dans l'industrie (groupe 3)}

Un autre groupe d'entreprises se distingue radicalement du précédent en ce sens qu'elles utilisent le plus largement possible aussi bien toutes les sources de connaissances basées sur la recherche (instituts et universités) que les consultants publics et privés. Elles sont aussi nombreuses à avoir des échanges avec d'autres petites entreprises, notamment dans le cadre de réseaux formels d'entreprises et coopèrent avec leurs clients et leurs fournisseurs. Dans ce groupe, les dirigeants sont très nombreux à avoir des responsabilités dans le cadre de leurs associations professionnelles.

Ces entreprises se distinguent aussi des autres par un niveau technologique en moyenne bien supérieur (le groupe comportant une majorité d'entreprises à haut niveau technologique) et une stratégie d'échanges technologiques élargis et différenciés, associant notamment plusieurs catégories de partenaires liés à la recherche. En ce qui concerne les instituts de recherche de branche, nos résultats montrent que les entreprises de ce groupe font un usage à la fois plus large et plus spécialisé de ces instituts, le tiers d'entre elles participant notamment à des projets de recherche en collaboration avec un institut. Cette stratégie d'échanges technologiques élargis rappelle fortement celle de cas étudiés antérieurement (Belotti, 1996, 1999a) et pour lesquels nous avons mis au jour les synergies opérées dans le temps par les entrepreneurs entre différents types de relations, pour développer et professionnaliser leurs échanges technologiques.

Une autre caractéristique des entreprises de ce groupe est leur ancrage dans les institutions propres à leur industrie, une très grande proportion des chefs d'entreprises du groupe ayant un engagement à l'échelle nationale dans les associations professionnelles. Â cela, il faut ajouter que ces entreprises déclarent, à un degré significativement plus élevé que les autres, participer aux activités de diffusion technologique des associations professionnelles et avoir elles-mêmes des activités de diffusion visant d'autres petites entreprises par le biais de visites reçues et de conférences dispensées. 


\subsection{Stratégie d'échanges ciblés et de complémentarité (groupe 4)}

Un troisième groupe d'entreprises coopère en grand nombre avec leurs clients sur les produits mais surtout avec leurs fournisseurs et équipementiers. Elles ont recours en moyenne à moins de sources privées ou institutionnelles de connaissances que le groupe précédent, en étant axées sur des sources plus spécifiques (instituts de recherche industrielle, même hors branche, et consultants privés en premier lieu) tout comme elles sont dans la plus grande proportion à avoir des échanges personnels spécifiques dans d'autres petites entreprises. À l'inverse du groupe précédent, leurs dirigeants ne sont pas beaucoup engagés dans les associations professionnelles. Ces entreprises ont un niveau technologique moyen assez élevé - plus de $80 \%$ des entreprises du groupe ayant un niveau technologique élevé ou moyen pour leur industrie. Le tableau 6 montre que ce qui les distingue des autres groupes, c'est la diversification des formations de leurs cadres. On peut ainsi penser que ces entreprises baseraient plutôt leur renouveau technologique sur cette compétence interne diversifiée en la complétant avec des échanges spécifiques et en adoptant une stratégie d'échange plus «individualiste » - à en juger par le très faible engagement des dirigeants au sein des institutions de l'industrie.

\subsection{Stratégie d'échanges plus limités et plus traditionnels (groupe 2)}

Un quatrième groupe d'entreprises apparaît moins ouvert que les deux groupes précédents à l'exploitation de sources privées ou institutionnelles de connaissances. Elles sont essentiellement axées sur la collaboration avec leurs clients pour le développement tant de leurs produits que de leurs procédés et avec d'autres entreprises dans le cadre de réseaux formels d'échange. La majorité des entreprises de ce groupe ont un niveau technologique moyen pour leur industrie. Elles sont en moyenne moins actives que les groupes précédents à développer leur compétence, le niveau moyen de celle-ci étant plus proche des entreprises du premier groupe que de celui des deux autres groupes. On peut donc parler d'entreprises plus traditionnelles dans leurs modes d'échanges technologiques, centrées sur un nombre limité de catégories de partenaires, les clients et les collègues d'autres petites entreprises.

\section{Discussion et conclusions}

Ainsi notre enquête révèle que les entreprises échangent des connaissances avec un large éventail de partenaires d'échange en liaison avec leur activité de développement technologique et confirme le caractère fortement interactif de ce développement mis en évidence dans les études empiriques antérieures. Elle révèle qu'une grande partie des entreprises collaborent avec leurs clients et leurs fournisseurs de matières premières et services pour développer leurs produits et procédés, mais nous n'avons pas trouvé de relation statistiquement significative entre la 
présence d'une telle coopération et le niveau technologique des entreprises. L'analyse typologique des données a montré qu'en fait une coopération technologique avec les clients et les fournisseurs n'est associée à un niveau élevé de développement technologique que dans les cas où les entreprises savent aussi mobiliser le support d'autres acteurs dans leur environnement, et notamment la recherche. Cela remet en cause la prééminence qui est donnée par de nombreux auteurs, de façon, à notre avis simplificatrice, aux relations clients-fournisseurs pour le développement technologique des PMI ( $c f$. Håkansson, 1989 ; Easton et Araujo, 1992). Les différences mises en évidence entre les deux branches concernant les modes d'échanges technologiques avec les clients et les fournisseurs démontrent que les marges de manœuvre des PMI à cet égard peuvent varier beaucoup d'un contexte industriel à l'autre. Nos résultats, issus d'un échantillon représentatif de PMI de deux industries, tendent à confirmer la thèse que nous avancions à l'issue d'études de cas faites antérieurement sur un petit nombre de PMI du bois technologiquement avancées (Belotti, 1996, 1999a et b) : une stratégie d'échanges élargis, en créant des synergies entre différents types d'échanges, augmente la capacité des PMI à négocier un échange technologique pertinent avec leurs clients. Cela est d'autant plus important à souligner que les PMI se trouvent souvent dans un rapport de pouvoir défavorable à l'égard de leurs clients, et notamment les plus exigeants d'entre eux.

Un autre point à souligner en conclusion concerne l'importance donnée par les entrepreneurs enquêtés aux canaux d'informations et d'échanges technologiques mis en place par leurs associations professionnelles de branche, bien que le rôle de ces organisations n'ait quasiment pas été abordé dans les études antérieures. Dans les deux industries suédoises étudiées, les organisations professionnelles créent de nombreux « espaces » d'échanges technologiques (réseaux, réunions, séminaires, etc.) jugés importants par les entreprises pour leur développement technologique. Non seulement nous montrons que les entreprises sont très nombreuses à se référer à ces espaces, mais aussi qu'un engagement dans ces organisations professionnelles et une activité de diffusion technologique caractérisent les entreprises à haut niveau technologique et ayant une stratégie d'échanges technologiques élargis. Encore une fois, ces résultats d'enquête vont dans le sens des analyses avancées à l'issue de nos études de cas antérieurs, où nous soulignons le rôle de la proximité « organisationnelle » (au sens de Bellet, Colletis et Lung, 1993) plutôt que géographique, dans le choix des partenaires d'échanges technologiques des entreprises étudiées (Belotti, 1996, 1999b). L'appartenance à une même branche englobe en effet de nombreuses dimensions de proximité entre les entreprises (proximité structurelle, organisationnelle, technologique, culturelle) dont nous avons auparavant signalé l'importance dans les logiques d'échanges de certains dirigeants d'entreprises (Belotti, 1999b) et qui fonderait la capacité des organisations professionnelles étudiées à créer et entretenir des «milieux innovateurs », au sens où l'a défini Camagni (1991). Notons par ailleurs que l'enquête (qui n'a certes pas été conçue pour tester 
l'importance de la proximité géographique dans les échanges technologiques, mais qui inclut des variables englobant cette dimension) ne met pas en évidence une importance particulière de la proximité géographique des partenaires dans les stratégies d'échanges technologiques des différentes entreprises. Elle ne fournit pas d'éléments appuyant l'idée que le milieu local (au sens géographique du terme) serait un élément «crucial » de support à l'innovation (comme l'affirme les théoriciens des « milieux innovateurs »). Nos résultats mettent donc cette fois en question la prééminence qui est donnée par certains auteurs à la proximité géographique et aux milieux locaux pour l'innovation et le renouveau technologique des entreprises. Ils suggèrent d'appuyer le support au développement technologique des PMI sur différentes dimensions de proximité selon les entreprises ciblées et de tenir compte du rôle de diffuseur que peuvent jouer certaines entreprises dans différents contextes institutionnels.

En ce qui concerne enfin les stratégies d'échanges que nous avons fait ressortir, nous pouvons les considérer comme représentant différents stades du processus de développement technologique pouvant être engagé sur le long terme par des PMI - même si nous pouvons penser que nombre d'entreprises du groupe 1 ont peu de chances de pouvoir relever à terme le défi du développement technologique et de la compétitivité. La typologie à laquelle nous avons abouti se rapproche ainsi de celle des compétences technologiques « en escalier » développée par exemple par Arnold et Guy (1996), chaque entreprise pouvant faire progressivement son chemin vers un haut niveau technologique en développant ses compétences et son réseau d'échange avec l'environnement.

Il convient cependant de discuter plus avant les deux stratégies (groupes 3 et 4) associées aux plus hauts niveaux techniques et de compétence. Même si la stratégie d'échanges des entreprises du groupe 4 (stratégie d'échanges ciblés) est associée à un niveau technologique dans les entreprises légèrement inférieur à celui des entreprises du groupe 3 (stratégies d'échanges élargis et ancrés dans l'industrie), nous sommes en droit de nous demander si elle n'est pas plus pertinente pour faire face, à long terme, à l'évolution d'une économie globalisée. Cette hypothèse se fonde sur les compétences plus diversifiées des entreprises du groupe 4 , sur la priorité qu'elles donnent au développement des compétences dans le domaine de l'organisation, du marketing et des achats et même aussi à leur position d' « outsider » par rapport aux institutions de la branche. Ces caractéristiques peuvent en effet correspondre davantage à une orientation vers l'innovation, par rapport aux autres entreprises, techniquement excellentes, plus fortement orientées sur l'amélioration de leur technologie de production et bien intégrées dans le fonctionnement des institutions de la branche.

Stymne (1999), à partir d'une étude européenne sur les PMI et les technologies de l'information, relève le même type de contraste parmi des entreprises innovantes suédoises et développe le même type de réflexion sur les aptitudes des 
deux types d'entreprises à exploiter les NTIC pour renforcer leur compétitivité à long terme. Notons aussi au passage que les deux types de stratégie identifiées dans les groupes 3 et 4 pourraient assez bien se rapprocher des « défensifs » et des « explorateurs » de la typologie de Miles et Snow (1978), les premiers se caractérisant par une grande compétence dans le domaine de l'activité de l'entreprise, les seconds étant plutôt perpétuellement à la recherche de nouvelles occasions d'affaires et alimentant le changement et l'incertitude au sein de leur branche. Aussi, si nous considérons le rôle que peut jouer chaque type d'entreprise au niveau de la branche, nous pouvons faire l'hypothèse que les entreprises caractérisées par leurs échanges technologiques élargies (groupe 3) tendraient plutôt à canaliser le paradigme stratégique dominant dans la branche en matière de développement technologique, alors que les entreprises du groupe 4 pourraient plutôt tendre à avoir un rôle régénérateur de la branche, en développant des stratégies en matière de technologie plus autonomes par rapport aux normes dominantes.

Ainsi, l'identification et la discussion de ces différentes stratégies d'échanges technologiques parmi les PMI peuvent-elles fournir quelques repères d'action aux acteurs engagés dans le support technologique aux PMI, notamment pour le ciblage de leurs initiatives. Les résultats posent en même temps des questions nouvelles qui peuvent trouver des réponses par le biais d'études de cas plus approfondies. La mise en évidence, par exemple, d'un recours assez large par ces petites entreprises traditionnelles aux organisations de recherche, demanderait une étude qualitative détaillée des échanges opérés avec ces acteurs. Il reste aussi de nombreuses connaissances à acquérir sur la manière dont chaque type d'entreprise utilise son environnement local, institutionnel et commercial pour soutenir son développement technologique et surtout sur les interférences ou synergies qu'elles créent entre ces différents types d'environnement dans leurs modes d'échanges.

\section{Bibliographie}

AllEN, T.J., D.B. HYMAN ET D.L. PINKNEY (1983), « Transferring technology to the small manufacturing firm: a study of technology transfer in three countries », Research Policy, vol. 12, p. 199-211.

ARNOLD, E. et K. GUY (1996), «New approaches and continued needs in innovation policy », dans Samverkan mellan högskolan och de små och medelstora företagen, SOU, 89, Stockholm, NYFOR-kommittén.

BEESLEY M. et R. ROTHWELL (1987), «Small firm linkages in the United Kingdom » dans R. Rothwell et J. Bessant (dir.), Innovation, Adaptation and Growth, Amsterdam, Elsevier.

BeLlet, M., G. Colletis et Y. LuNG (1993), Introduction au numéro spécial «Économie de proximités », Revue d'Économie Régionale et Urbaine, $\mathrm{n}^{\circ}$ 3, p. 357-361.

BELOTTI, C. (1995), «Technological renewal in small firms : the nature and role of linkages between large and small firms », Frontiers of Entrepreneurship Research, p. 667-679. 
BELOTTI, C. (1996), Teknikförnyelseprocesser i små företag. Om företagens samspel med omgivningen, Thèse 21, Uppsala, Sveriges lantbruksuniversitet, Institutionen för ekonomi.

BELOTTI, C. (1997), «Sweden - evaluation of technical support for SMEs », Report from TAFTIE-conference, novembre 1996, Stockholm, NUTEK.

BELOTTI, C. (1999a), « Technological renewal in small manufacturing enterprises », dans B. Johannisson et H. Landström (dir.), Images of Entrepreneurship and Small Business : Emergent Swedish Contributions to Academic Research, chapitre 8, Lund, Studentlitteratur.

BELOTTI, C. (1999b), «La modernisation technologique des petites entreprises en zone rurale : Réflexions autour d'études de cas suédois », Revue internationale PME, vol. $12, \mathrm{n}^{\text {os }} 1-2$.

BELOTTI, C. et C. TUNÄLV (1998), « Acquisition of technological knowledge in small and medium-sized manufacturing companies in Sweden », The International Journal of Technology Management, vol. 18, $\mathrm{n}^{\text {os }} 3-4$, p. 353-371.

Callon, M. (1991), «Réseaux technico-économiques et irréversibilité », dans R. Boyer et al. (dir.), Les formes de l'irréversibilité en économie, Paris, EHESS.

CAMAGNI, R. (1991), «Local "Milieu", Uncertainty and innovation networks - towards a new dynamic theory of economic space», dans R. Camagni (dir.), Innovation Networks : Spatial Perspectives, Londres, Belhaven Press.

CARlsson, B. et R. STANCKIEWICZ (1991), "On the nature and composition of technological systems », Journal of Evolutionary Economics, vol. 1, p. 93-118.

EASTON, G. et L. ARAUJO (1992), « Non-economic exchange in industrial networks », dans B. Axelsson et G. Easton (dir.), Industrial Networks : A New View of Reality, Londres, Routledge, p. 62-84.

FÖLSTER, S. (1991), «Hinder för teknikspridning i Sverige», dans Forskning, teknikspridning och produktivitet, Expertrapport 10, Stockholm, Produktivitetsutredningen.

GASSE, Y. (1986), «Le processus d'adoption des nouvelles technologies par les PME», dans P.-A. Julien, A. Joyal et J. Chicha (dir.), La PME en mutation, Sainte-Foy, Presses de l'Université du Québec.

GASSE, Y. (1995), « The technological innovation process : a matter of entrepreneurship for small firms », présenté au Babson College Entrepreneurship Research Conference, Londres, 9-13 avril.

GROTZ, R. et B. BRAUN (1996), «Innovation et intégration régionale : le rôle des réseaux manufacturiers dans l'industrie allemande d'ingénierie mécanique », Revue internationale PME, vol. 9, $\mathrm{n}^{\text {os }} 3-4$, p. 37-66.

GUEDJ, B. et F. PICARD (1994), « Pratiques innovatrices des PME rhône-alpine : une approche par la diversité des sources de l'innovation technologique », Revue internationale $P M E$, vol.7, $\mathrm{n}^{\text {os }} 3-4$, p. 41-64.

HÉRAUD, J.-A. et C. NANOPOULOS (1994), «Les réseaux de l'innovation dans les PME : illustration sur le cas de l'Alsace », Revue internationale $P M E$, vol. 7, $\mathrm{n}^{\text {os }} 3-4, \mathrm{p} .65-86$.

HolMQUIST, C. et E. SUNDIN (1997), «In search of excellens - eller att falla i farstun för den utländska », dans Programme and abstracts, 14th Nordic Conference on Business Studies, août 1997 dans Bodö, Bodø Graduate School of Business, Bodø. 
HÅKANSSON, H. (1989), Corporate Technological Behaviour : Co-operation and Networks, Londres, Routledge.

HÖGLUND, L. et O. PERSSON (1984), « Kontaktmönster bland svenska tekniker », Rapport 23, Umeå, Inforsk.

JULIEN, P.-A. (1996), «Entrepreneuriat, développement du territoire et appropriation de l'information ", Revue internationale PME, vol. 9, $\mathrm{n}^{\text {os }} 3-4$, p. 149-178.

JULIEN, P.-A. et L. HÉBERT (1986), «Le rythme de pénétration des nouvelles technologies dans les PME manufacturières », Journal of Small Business and Entrepreneurship, vol. 3 , no 4 , p. 24-35.

JULIEN, P.-A., J.-B. CARRIÈRE et L. HÉBERT (1988), «Les facteurs de diffusion et de pénétration des nouvelles technologies dans les PME québéquoises », Revue internationale PME, vol. 1, n' 2, p. 193-222.

Julien, P.-A., J.-B. CARrière, L. RAYMOnD et R. LAChance (1994), « La gestion du changement technologique dans la PME manufacturière au Québec: une analyse de cas multiples », Revue internationale $P M E$, vol. 7, $\mathrm{n}^{\text {os }} 3-4$, p. 87-121.

LANGLEY, A. et J. TRUAX (1994), «A process study of new technology adoption in smaller manufacturing firms », Journal of Management Studies, vol. 31, $\mathrm{n}^{\circ} 5$.

LAPOINTE, S. (1991), «L'adoption de nouvelles technologies dans le secteur du vêtement », Étude du processus informationel, présenté à ISCB-conférence, Trois-Rivières, Université du Québec à Trois-Rivières.

LE BAS, C. et I. GÉNIAUX (1994), «Le management des relations technologiques et les PME», Économies et Sociétés, Série Sciences de gestion.

Leijon, S., F. Norrgren, S. Rubenowitz, B. Rundblad et A. TARgama (1988), Den nya tekniken : Förändring eller anpassning, Göteborg, BAS.

LEONARD-BARTON, D. (1982), Swedish Entrepreneurs in Manufacturing and Their Sources of Information, Boston, Center for Policy Applications, MIT.

MARChESNAY, M. (1994), «Le management stratégique », dans P.-A. Julien (dir.), Les PME, bilan et perspectives, Paris, Economica, p. 133-162.

MILES, R.E. et C.C. SNOw (1978), Organizational Strategy, Structure and Process, New York, McGraw-Hill.

MøNSTED, M. (1989), «Small enterprises coping with the challenges of information technology », dans F. Borum et P.H. Kristensen (dir.), Technological Innovation and Organizational Change: Danish Patterns of Knowledge, Networks and Culture, Copenhagen, Copenhagen Business School.

NORÉN, L. (1987), Ny teknik i småföretag från idé till användning, Göteborg, BAS, Handelshögskolan vid Göteborgs universitet.

ROGERS, E. M. (1983), Diffusion of Innovations, $3^{\mathrm{e}}$ édition, New York, Free Press.

Rothwell, R. et M. DodgSON (1991), «External linkages and innovation in small and medium-sized enterprises », $R$-D Management, vol. $21, \mathrm{n}^{\circ} 2$.

SMall Business Research Center (1992), The State of British Enterprise, Cambridge, Cambridge University, Department of Applied Economics.

STYMNE, B. (1999), « COMPETE results in Sweden », dans Farizelli (dir.), Innovating SME Business Practices, Bologna, Edizioni Pendragoni/Nomisma. 